\title{
Gene expression profiling of metastatic brain cancer
}

\author{
VAHE MICHAEL ZOHRABIAN ${ }^{1}$, HARI NANDU ${ }^{1}$, NICHOLAS GULATI ${ }^{1}$, GREG KHITROV ${ }^{3}$, \\ CONNIE ZHAO $^{3}$, AVINASH MOHAN ${ }^{1}$, JOSEPH DEMATTIA ${ }^{1}$, ALEX BRAUN ${ }^{2}$, \\ KAUSHIK DAS $^{1}$, RAJ MURALI ${ }^{1}$ and MEENA JHANWAR-UNIYAL ${ }^{1}$ \\ Departments of ${ }^{1}$ Neurosurgery and ${ }^{2}$ Pathology, New York Medical College, \\ Valhalla, NY 10595; ${ }^{3}$ The Rockefeller University, New York, NY 10021, USA
}

Received March 16, 2007; Accepted April 23, 2007

\begin{abstract}
Gene expression profiling of metastatic brain tumors from primary lung adenocarcinoma, using a $17 \mathrm{k}$-expression array, revealed that 1561 genes were consistently altered. Further functional classification placed the genes into seven categories: cell cycle and DNA damage repair, apoptosis, signal transduction molecules, transcription factors, invasion and metastasis, adhesion, and angiogenesis. Genes involved in apoptosis, such as caspase 2 (CASP2), transforming growth factor- $\beta$ inducible early gene (TIEG), and neuroprotective heat shock protein 70 ( $H s p 70)$ were underexpressed in metastatic brain tumors. Alterations in Rho GTPases (ARHGAP26, ARHGAP1), as well as down-regulation of the metastasis suppressor gene KiSS-1 were noted, which may contribute to tumor aggression. Overexpression of the invasion-related gene neurofibromatosis 1 (NF1), and angiogenesis-related genes vascular endothelial growth factor-B $(V E G F-B)$ and placental growth factor $(P G F)$ was also evidenced. Brain-specific angiogenesis inhibitors 1 and 3 (BAI1 and BAI3) were underexpressed as well. Examination of cell-adhesion and migration-related genes revealed an increased expression of integrins and extracellular matrices collagen and laminin. The study also showed alterations in p53 protein-associated genes, among these increased gene expression of p53, up-regulation of Reprimo or candidate mediator of the p53-dependent G2-arrest, down-regulation of p53-regulated apoptosis-inducing protein 1 (p53AIP1), decreased expression of tumor protein inducible nuclear protein 1 (p53DINP1), and down-regulation of Mdm4 $(M D M X)$. The results demonstrated that genes involved in adhesion, motility, and angiogenesis were consistently upregulated in metastatic brain tumors, while genes involved in
\end{abstract}

Correspondence to: Dr Meena Jhanwar-Uniyal, Departments of Neurosurgery and Experimental Pathology, New York Medical College, Valhalla, NY 10595, USA

E-mail: meena_jhanwar@nymc.edu

Key words: gene expression profiling, metastatic brain tumor, p53, neurofibromatosis 1, Rho GTPase, vascular endothelial growth factor apoptosis, neuroprotection, and suppression of angiogenesis were markedly down-regulated, collectively making these cancer cells prone to metastasis.

\section{Introduction}

Metastasis, the process by which cells break free from a primary tumor, enter the bloodstream or lymphatic system, and spread to distant sites within the body, remains a major cause of morbidity and mortality in many malignant neoplasms. Breast, lung, and ovarian cancer patients may develop metastatic lesions in the brain, often associated with a poor prognosis given that the highly specialized and delicate microenvironment of the brain is disrupted. To metastasize to the brain, cancer cells must attach to microvessel endothelial cells and invade the blood-brain barrier (BBB). After passing through the $\mathrm{BBB}$, a cell clone must attach to an area where angiogenesis can take place. The metastatic phenotype characterizes cells with the ability to migrate from the primary tumor, survive in the circulation, pass through the BBB, invade distant tissue, and form blood vessels needed for growth and proliferation.

Metastasis is a complication of neoplastic disease that renders a poor prognosis. Central nervous system metastases continue to afflict those with systemic cancer, and their incidence in the population is most likely underestimated due to differences in methods of assessment (1). Brain metastases frequently involve an area right beneath the gray-white matter junction, and the largest percentage of these lesions involve the cerebrum, with cerebellum and brainstem lesions collectively accounting for only $20 \%$ (1). A majority of brain metastases arise from lung or breast cancer cells that travel hematogenously, although testicular and melanoma cancers pose the greatest risk for brain involvement (1). Lung cancer metastases to the brain are also frequently multiple (52\%) (1). Of all known primary tumors that metastasize to the brain, lung cancer is characterized by the shortest duration from diagnosis of the primary cancer to brain involvement ( 3 months), as well as the shortest duration from metastasis to death (3 months, small-cell lung cancer), thus underscoring the aggressive nature of this devastating disease (1). Elucidating the molecular and biological basis of lung cancer metastasis to the brain may allow future therapy to be targeted at preventing hematogenous dissemination of malignant lung cancer cells. 
The exact genetic and/or epigenetic changes that cause cancers to become metastatic remain elusive. The prevailing model of metastasis holds that most primary tumors have a low metastatic potential; however, rare cells within the primary tumors may acquire metastatic capacity through somatic mutations (2). This view was challenged by studies that revealed that metastasis-prone gene expression is present in primary tumors of patients and can predict clinical outcome (3). Studies have attempted to profile subsets of genes that modulate the metastatic phenotype. In one particular study, a set of genes mediating breast cancer metastasis to the lung was discovered to encode mostly for extracellular products such as HER/ErbB growth factor receptor ligand epiregulin, chemokines such as CXCL1, cell adhesion receptors, and proteases (4). Another study also provided evidence that discrete sets of genes are associated with tissue-specific metastasis (5). Several studies have provided evidence that certain genes, such as p53, may be involved in brain metastases $(6,7)$. Since the discovery of microarray technology, attempts have been made to identify the molecular markers associated with metastasis $(3,4)$. In that, organ-specific metastasis has been associated with a set of genes that are involved in functions such as angiogenesis, invasion, metastasis, and tumorigenesis. Investigators have found that cells originating from the same organ may have discrete genetic signatures that may encode for metastases to different specific organs (5). There is no study, thus far, that represents the genes associated with cancer metastasis to the brain. Herein, we identify genes expressed in metastatic brain tumors from primary adenocarcinoma of the lung.

\section{Materials and methods}

Materials. Tissues from brain metastasis of primary adenocarcinoma of the lung (two patients: Following HIPPA regulation) were compared with non-metastatic lung tissue.

Isolation of total cellular RNA. Total RNA was extracted using TRIzol Reagent (Life Technologies, Inc.). Isolated RNA was electrophoresed through $1.0 \%$ agarose-formaldehyde gels to verify the quality. The concentration and purity of RNA were determined from absorbance measurements at 260 and $280 \mathrm{~nm}$.

cDNA synthesis and microarray hybridization. Total cellular RNA $(7 \mu \mathrm{g})$ was annealed to oligo(dT) and reverse-transcribed in the presence of Cy3-labeled or of Cy5-labeled dUTP (Amersham Pharmacia Biotech, Piscataway, NJ), using 10,000 units/ml of Superscript II reverse transcriptase (Life Technologies, Inc.). The resulting Cy3- and Cy5-labeled cDNAs were treated with RNase One (Promega Corporation, Madison, WI) for $10 \mathrm{~min}$ at $37^{\circ} \mathrm{C}$, combined, and purified by passing through a Centricon-50 filtration spin column (Millipore, Bedford, MA). Hybridizations were performed on cDNA microarray glass overnight at $50^{\circ} \mathrm{C}$ in a humidified chamber. Each slide contained 17,000 unique human cDNA clones.

Scanning, gridding, and analysis. Slides were scanned using a microarray scanner Scan Array Lite (Perkin Elmer, Waltham, MA). The scanner output tif images were gridded, and the fluorescence intensities of microarrays were
Table I. List of primers used for RT-PCR to validate the expression of selected genes from cDNA microarray analysis.

\begin{tabular}{llc}
\hline Gene & \multicolumn{1}{c}{ Primers } & $\begin{array}{c}\text { Product } \\
\text { (bp) }\end{array}$ \\
\hline MMP-1 & F: 5' TGTGGTGTCTCACAGCTTCC 3' & 250 \\
& R: 5' CACATCAGGCACTCCACATA 3' & \\
a3-integrin & F: 5' TATTCCTCCGAACCAGCATC 3' & 250 \\
& R: 5' CTCTTCATCTCCGCCTTCTG 3' & \\
VEGF & F: 5' CCTTGCTGCTCTACCTCCAC 3' & 197 \\
& R: 5' CACACAGGATGGCTTGAAGA 3' & \\
Rho GTPase & F: 5' TTGTCCTTGGGGAGTACAGG 3' & 160 \\
& R: 5' GAGAGCCCAGCGAAGTTATG 3' & \\
Fibronectin & F: 5' ACCAACCTACGGATGACTCG 3' & 230 \\
& R: 5' GCTCATCATCTGGCCATTTT 3' & \\
PTEN & F: 5' AGACCATAACCCACCACAGC 3' & 254 \\
& R: 5' ACACATAGCGCCTCTGACTG 3' & \\
B-actin & F: 5' TCACCCACACTGTGCCCATCTACGA 3' & 295 \\
& R: 5' CAGCGGAACCGCTCATTGCCAATGG 3' & \\
\hline
\end{tabular}

calculated using the Iobion Genetraffic software. Final intensities of green and red channels were filtered and the ratios of the red intensity to the green intensity were determined. The average expression level of each gene in two groups was calculated, and the cutoff value was set to 2fold up or down after normalization of data.

RNA expression using RT-PCR. Gene expression was determined by RT-PCR with a One-Step RT-PCR kit (Qiagen, CA) using primers for specific genes designed by using Primer3 (MIT, MA) and are shown in Table I and Fig. 1.

\section{Results}

The gene expression profile of metastatic brain tumors originating from adenocarcinoma of the lung was compared to that of non-malignant lung RNA utilizing cDNA microarrays. The expression profile of genes under different pathways/ functions revealed the possible involvement of some known, but several unknown, genes in the metastasis of peripheral tumors to the brain and is presented in Table II and Fig. 2.

Of the 17,000 genes analyzed in each experiment, $~ 5.6 \%$ were ESTs. The cDNA microarray results revealed 1561 genes, the expressions (over and underexpressed) of which were altered significantly and consistently between metastatic and benign tumor samples. The number of overexpressed genes was 1295 and the underexpressed genes were 266 . To extrapolate an association between these differentially expressed genes and various functional roles associated with cancer metastasis, we grouped genes of significance into seven categories based on function, namely, cell cycle and DNA damage repair, apoptosis, signal transduction molecules, transcription factors, invasion and metastasis, adhesion, and angiogenesis (Table I). This analysis represents gene alterations present in both tumor samples. Certain important genes 


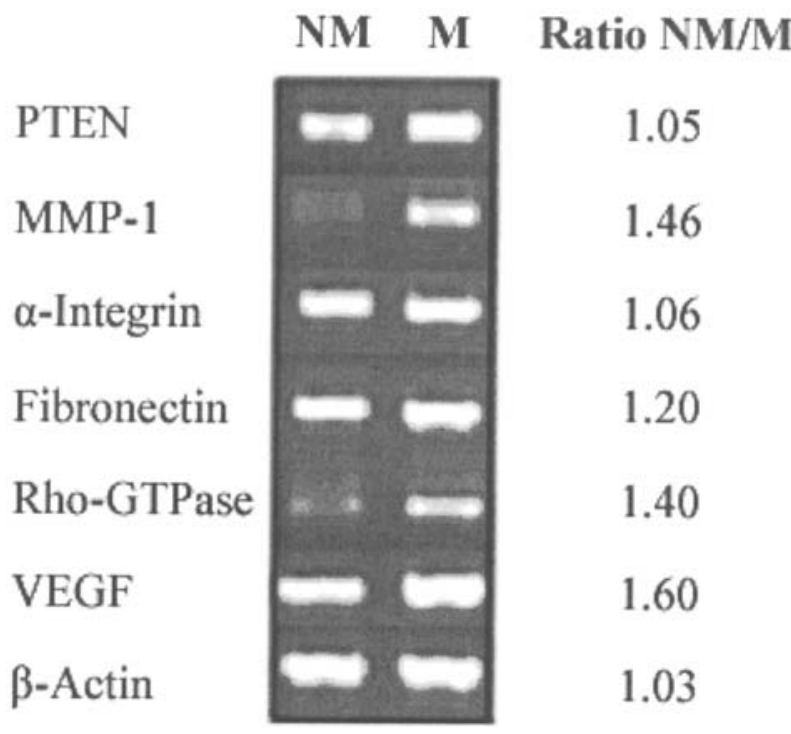

Figure 1. Semi-quantitative analysis of genes in metastatic brain cancer. Confirmation of gene expression of selected genes by RT-PCR was performed on RNA extracted from non-metastatic (NM) lung adenocarcinoma and metastatic brain tumor tissue $(M)$. The ratio $(\mathrm{NM} / \mathrm{M})$ indicates the percentage difference between metastatic and non-malignant tissue (i.e. VEGF NM/M ratio of 1.60 translates to $60 \%$ overexpressed in metastatic brain tissue). associated with invasion and metastasis (PTEN, MMP1), adhesion (integrin $\alpha 3$ and fibronectin1), angiogenesis (VEGF) and cell migration (Rho GTPase) in these tumor samples were validated by RT-PCR.

Genes involved in apoptosis, such as CASP2, were found to be underexpressed. Additionally, heat shock protein $H s p O$, a major inducible heat shock protein known to inhibit cytochrome-C released apoptosome formation and caspase activation, was down-regulated. Rho GTPase activating protein ARHGAP26 and Rho guanine nucleotide exchange factor RhoGEF were underexpressed, while Rho GTPase activating protein $A R H G A P 1$ was up-regulated. Furthermore, decreased expression of EGFR and a strong overexpression of the ERBB3 gene was seen. The expression of $N F 1$ appeared to be higher in the metastatic brain tumors. Transcription factors $N F-\kappa B 2$ and GATAl were also up-regulated.

Adhesion molecules such as collagen XVI $\alpha 1$ (COL16A1), laminin $\beta 4$ (LAMB4), and integrins 2 and 6 (ITGB2 and $I T G B 6)$ were overexpressed. On the other hand, certain integrins such as ITG $\alpha 5, I T G \alpha 8$, and ITG $\beta 4$ were underexpressed. Significant underexpression of the metastasissuppressor gene KiSS-1 and tumor-repressor gene TIEG was observed. However, another metastasis-suppressor gene, $n m 23$, was underexpressed in only one tumor sample. Growth factors and angiogenesis-associated genes such as VEGF-A, $V E G F-B$, and $P G F$ were markedly up-regulated. Although

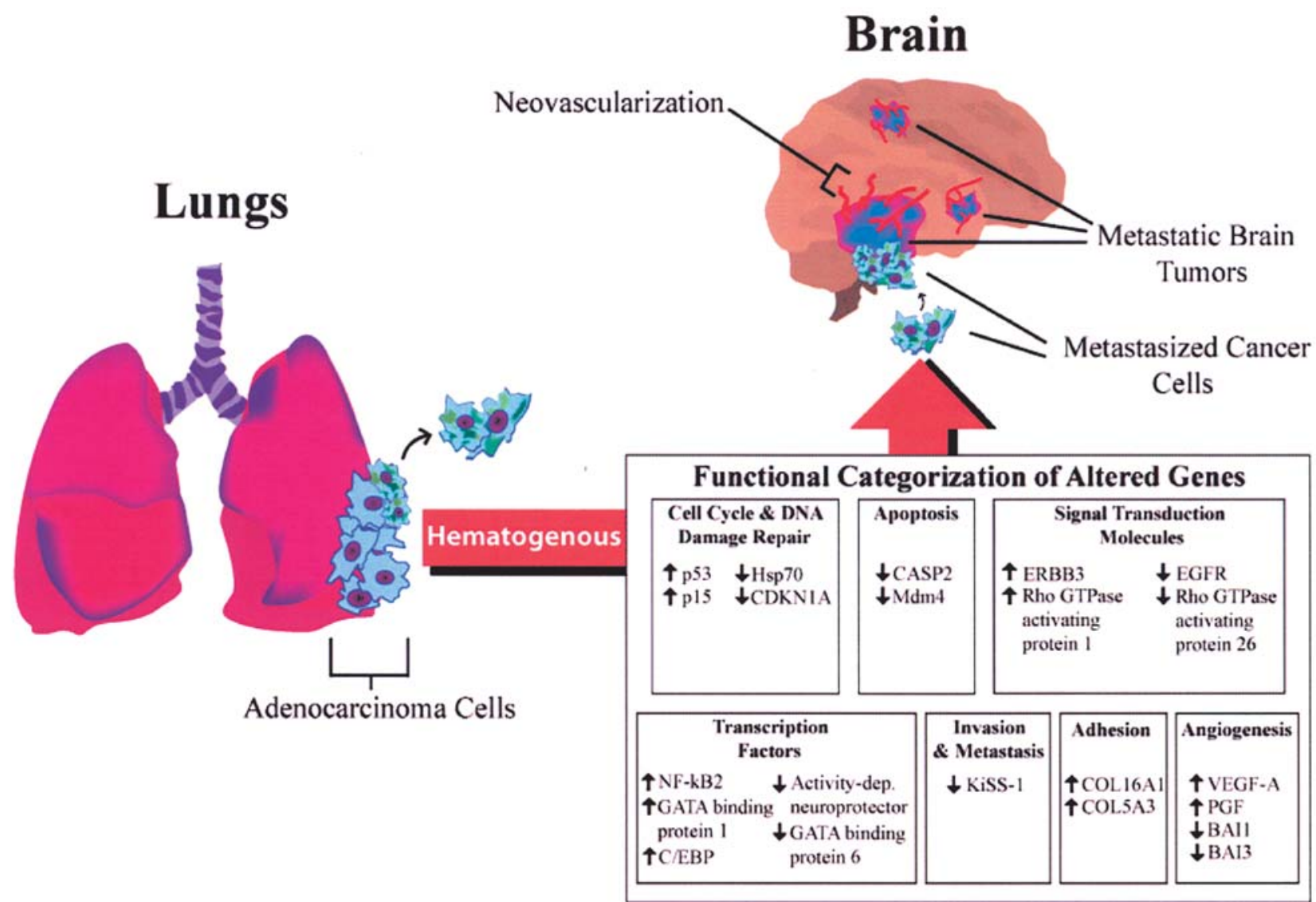

Figure 2. Representation of certain genes associated with lung adenocarcinoma metastasis to the brain. Shown here is the process of metastatic prone cells detaching from the primary site, entering the circulation, migrating to the brain, and forming new blood vessels. Also shown is a table categorizing some altered genes according to their functional attributes and their over and underexpressions based on up and down arrows, respectively. 


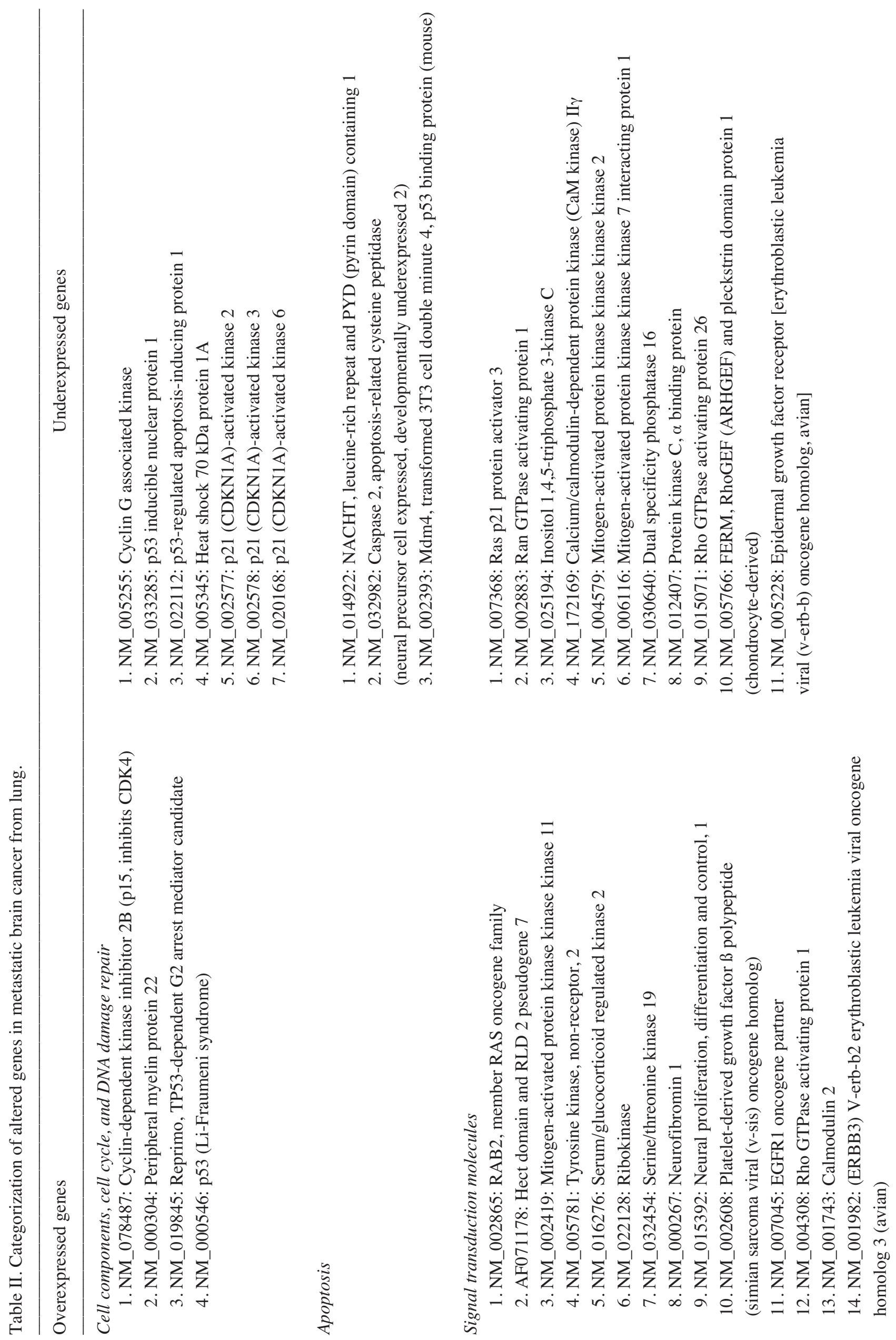




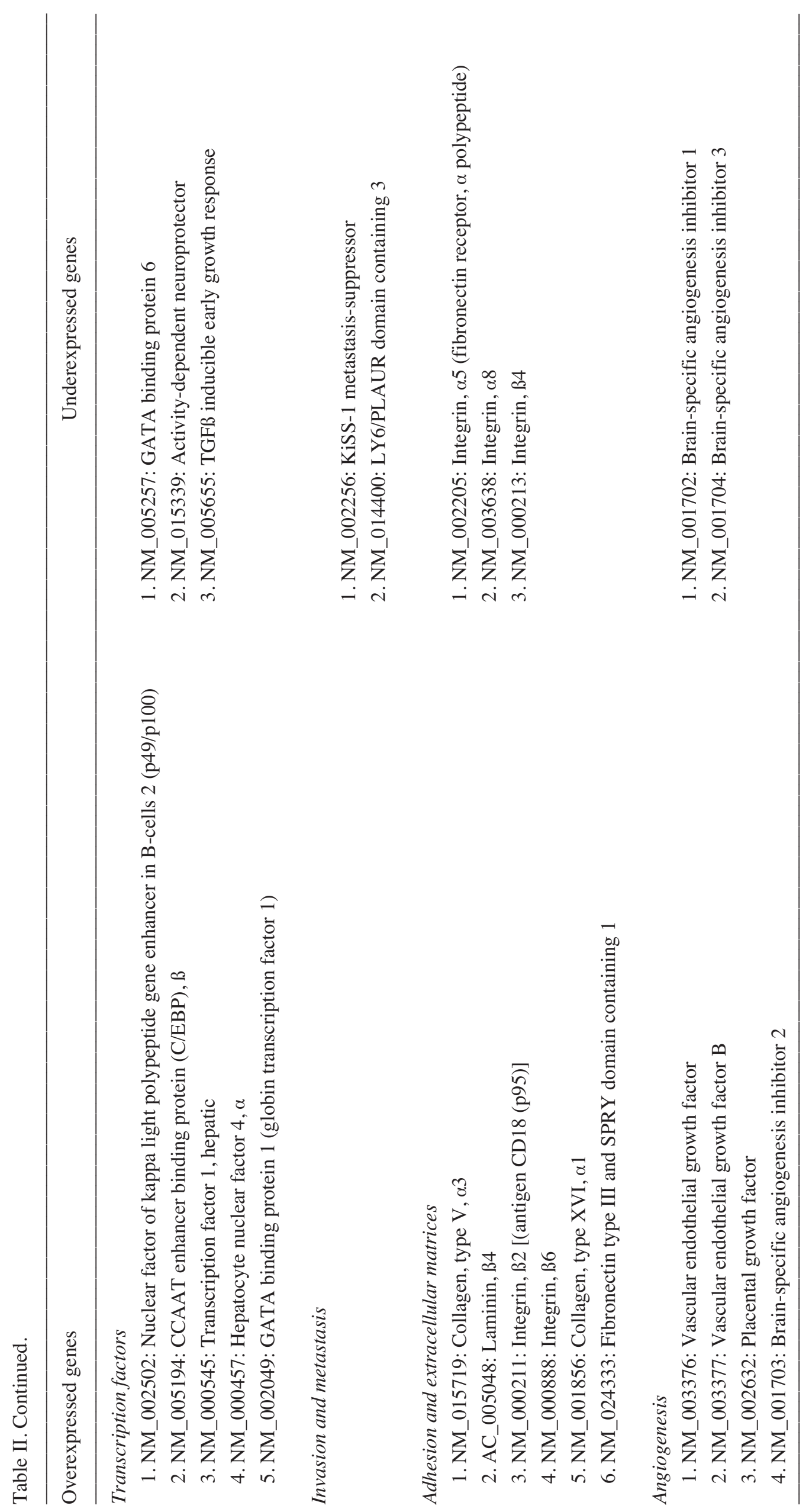


gene expression for brain-specific angiogenesis inhibitor 2 (BAI2) was augmented, BAII and BAI3 expression was suppressed.

\section{Discussion}

The metastatic brain tumors in our study revealed the downregulation of genes involved in apoptosis, such as caspase 2 (CASP2). Cysteinyl-directed aspartate-specific proteases (caspases) activate a cascade that culminates in apoptosis. Caspases 2, 8, 9, and 10 are classified under the family of initiator caspases, which cleave effector caspases to cause cell death. Caspase 2 induces neuronal cell death under conditions of growth factor depletion, B-amyloid toxicity, and through activation of Fas ligand and tumor necrosis factor $\alpha$ receptor (8). Therefore, the down-regulation of $C A S P 2$ in metastatic brain tumors may allow these tumor cells to bypass neuronal cell death pathways and may help promote tumorigenesis.

The Rho family of small monomeric GTPases, which includes RhoA, Rac1, and Cdc42, is involved in a multitude of cellular events, including cell cycle progression, cytoskeletal reorganization, and cell motility. RhoA activation is linked to the formation of focal adhesion complexes and actin stress fibers (9), while Rac1 and Cdc42 are associated with lamellipodia and filopodia formation, respectively. A recent investigation into Rho in lung cancers found that E1AF/PEA3, an Ets family transcription factor is often overexpressed in non-small cell lung carcinomas, activates the Rho/ROCK signaling pathway, lending to increased cell motility and metastasis (10). Also, a separate study utilized siRNA against the Rho proteins Rac1 and Rac2, and demonstrated that blocking the activity of Rac resulted in inhibition of lamellipodia, migration, and invasion of glioma and breast carcinoma cells (11). In line with these studies, our cDNA microarray analysis revealed a marked down-regulation of levels of ARHGAP26, a Rho GTPase activating protein (GAP), and the overexpression of ARHGAPl in both brain tumor samples. GAPs stimulate the conversion of Rho GTP to its inactive GDP-bound form, thus disallowing the effects of Rho on cell migration and growth. ARHGAP26 binds to the carboxy-terminus of focal adhesion kinase (FAK), a mediator of integrin-dependent cell signaling that promotes cell proliferation (12) and cell migration (13) intracellularly, and stimulates hydrolysis of RhoA and Cdc42. Furthermore, when injected into cells, ARHGAP26 results in a clearing of actin stress fibers that are normally linked to cell migratory ability. Given the release of the GAP's control over Rho GTPase activity, one expects a pronounced increase in all cell functions attributable to Rho GTPase proteins, such as metastasis, including regulation of tumor cell growth and metastasis in vivo (14). While up-regulation of $A R H G A P 1$ within the same brain tumor samples remains to be understood, it is possible that $A R H G A P 1$ is unique from other Rho GAPs such that it participates in a negative feedback loop that attempts to curb uncontrolled Rho GTPase activity within the metastasized brain samples. This may also explain down-regulation of RhoGEF in our microarray analysis, a Rho guanine nucleotide exchange factor that acts to activate the Rho family proteins by converting to GTP bound form.
Overexpression of EGFR family of receptor tyrosine kinases has been reported in many cancers. Studies have examined the differences in EGFR expression between primary and secondary glioblastoma multiforme (GBM), and have found that EGFR overexpression is associated with tumor growth and angiogenesis, as evidenced by the upregulation of genes encoding $V E G F, E T(B), P T N$ and receptor PTRPZ1, and Bax inhibitor 1 (15). Alternatively, our study revealed a down-regulation of EGFR in metastatic brain samples that poses a challenge in being understood. However, the analysis of biopsies of primary and secondary glioblastomas by Watanabe et al makes a clear case for the differential expression of p53 mutations, p53 protein accumulation, and EGFR overexpression in primary and secondary GBM (16). Hence, given the results of our study, it may appear that EGFR overexpression, seen in lung adenocarcinomas and other invasive tumors, is not required for established malignant phenotypes, while needed for the initial steps of invasion, and may be lost after cells have established a foothold in the 'subsoil' of the brain, then assuming a genotype more akin to primary gliomas. Alternatively, our samples showed a strong up-regulation of the ERBB3 gene, and a recent study that examined breast cancer metastasis found that ERBB3 signaling, through formation of a heterodimer with ERBB2, was strongly overexpressed in breast cancer metastasis to lung (1). Another study performed RT-PCR on primary tumor samples of patients with a high expression level of EGFR, ERBB2, and ERBB3 in non-small cell lung cancer, showing that increased ERBB3 levels most closely associated with shorter time to death (18).

NF1 was overexpressed in lung adenocarcinomas metastasized to the brain. Gene sequence analysis has revealed homology of NF1 with Ras GTPase-activating protein, and as such, the clinical manifestations of neurofibromatosis type 1 have been linked to loss of the GTPase activity, or accordingly, increased Ras activity $(19,20)$. NF1 regulates cell motility and invasion via activation of the Rho-ROCK-LIMK2 pathway, and subsequent phosphorylation of cofilin associated with actin stress fiber formation (21). However, our study showed increased expression of NF1 in metastatic brain tumors, which may indicate its interactions with other oncogenic molecules, a possible result of depletion of the NF1 gene product. For example, one study discovered a novel neurofibrominassociating protein, 14-3-3, which is directed towards the intracellular carboxy-terminus of neurofibromin and acts as a negative regulator of $N F 1$ by disabling $N F 1$ interaction with Ras-GTP, both in vitro and in vivo (22). Also, overexpression of NF1 induces FAK activation (23), the overexpression of which has been shown in cerebral metastases (24). In concordance with this, angiopoietin 2 stimulates MMP-2 expression lending to glioma cell invasion through the FAK signaling pathways (25), and thus, overexpression of NF1 may contribute to cancer metastasis through FAK.

The results of this study revealed up-regulation of $N F-\kappa B 2$ and GATA binding protein 1, and down-regulation of TIEG and GATA binding protein 6. p53 mutant tumor cells have been shown to strongly activate $N F-\kappa B 2$ (26), as $N F-\kappa B 2$ promotes cell survival through induction of the anti-apoptotic molecule Bcl-2 (2). Thus, $N F-\kappa B 2$ overexpression may indicate an oncogenic-like function. TIEG encodes a nuclear 
transcription factor regulated by $T G F-\beta$ that is involved in breast cancer metastasis to bone via metastasis genes ILII and $C T G F$ (5). Our finding also coincides with another study that examined the expression of TIEG in normal and malignant breast tissue and found the more invasive phenotype to be associated with lower TIEG levels (28).

Adhesion and adhesion molecules also play an important role in tumor progression. Our brain specimens revealed alterations in collagens, laminins, and integrins, specifically an increased expression of COL16A1, which is overexpressed in $U 33 M G$ glioma cells that are highly migratory and invasive (29). Our study also revealed down-regulation of ITGA8, which is influenced by nm23, a non-metastatic gene, which has been shown to down-regulate ITGA 8 in metastatic breast cancer cells (30). However, in our study, only one of the samples showed appreciable down-regulation of this gene.

The process of angiogenesis is an important event that contributes to the metastatic process. Our study showed an overexpression of $V E G F-A$ in metastatic brain tumors that has been associated with breast cancer metastases to brain in nude mice (31). In addition, in this study, up-regulation of $V E G F-B$ as well as $P G F$ was noted, both of which selectively act on the VEGFR-1 receptor to promote angiogenesis $(32,33)$. VEGF- $B$ is shown to promote and regulate adult neurogenesis (34), while expression of $P G F$ is associated with the aggressive and metastatic nature of small cell lung cancers (35). Furthermore, $P G F$ expression is up-regulated in primary tumors, among them gliomas, meningiomas, and schwannomas (36). Moreover, this study revealed decreased expression of BAII and BAI3 that suppress vascularization and may be involved in the periphery-to-brain metastasis sequence. Furthermore, BAII is p53-inducible and decreases stromal vascularization in pulmonary adenocarcinoma specimens (37).

Several p53-associated genes were also altered in our study, lending evidence to the role of mutant p53 in brain metastases (38). The $\mathrm{p} 53$ protein is a transcription factor that plays a vital role in regulating cell growth, DNA repair, and apoptosis in response to stressful conditions. Noticeable alterations in $\mathrm{p} 53$-associated genes included increased gene expression of p53, up-regulation of Reprimo or candidate mediator of the p53-dependent G2-arrest gene, known to arrest cells in the G2 phase of the cell cycle through involvement of cyclin B and the Cdc2 pathway, and down-regulation of p53regulated apoptosis-inducing protein 1 (p53AIP1), a novel p53 target that mediates apoptosis. Moreover, the study revealed decreased expression of tumor protein inducible nuclear protein 1 (p53DINP1) that is involved in p53-dependent apoptosis, as well as down-regulation of Mdm4 (MDMX) expression, a negative regulator of $\mathrm{p} 53$.

In conclusion, our study demonstrates that those genes involved in cell survival, neuroprotection, and suppression of vascularization, which were markedly underexpressed, make metastasis prone cells resistant to apoptosis. On the other hand, those overexpressed genes involved in motility, adhesion, and angiogenesis may impart a certain molecular signature that achieves functions necessary for the migratory and invasive phenotype of metastatic brain tumors (Fig. 2).

\section{Acknowledgements}

Part of this study was supported by funds from the American Research Foundation.

\section{References}

1. Brem S and Panattil JG: An era of rapid advancement: diagnosis and treatment of metastatic brain cancer. Neurosurgery 57 (Suppl 5): S5-S9, 2005.

2. Fidler IJ: Seed and soil revisited: contribution of the organ microenvironment to cancer metastasis. Surg Oncol Clin N Am 10: 257-259, 2001.

3. Ramaswamy S, Ross KN, Lander ES and Golub TR: A molecular signature of metastasis in primary solid tumors. Nat Genet 33: 49-54, 2003.

4. Minn AJ, Gupta GP, Siegel PM, Bos PD, Shu W, Giri DD, Viale A, Olshen AB, Gerald WL and Massague J: Genes that mediate breast cancer metastasis to lung. Nature 436: 518-524, 2005.

5. Kang Y, Siegel PM, Shu W, Drobnjak M, Kakonen SM, Cordon-Cardo C, Guise TA and Massague J: A multigenic program mediating breast cancer metastasis to bone. Cancer Cell 3: 537-549, 2003.

6. Arnold SM, Young AB, Munn RK, Patchell RA, Nanayakkara N and Markesbery WR: Expression of p53, bcl-2, E-cadherin, matrix metalloproteinase-9, and tissue inhibitor of metalloproteinases- 1 in paired primary tumors and brain metastasis. Clin Cancer Res 5: 4028-4033, 1999.

7. Schlegel U, Rosenfeld MR, Volkenandt M, Rosenblum M, Dalmau $\mathrm{J}$ and Furneaux H: p53 gene mutations in primary lung tumors are conserved in brain metastases. J Neurooncol 14: 93-100, 1992.

8. Troy CM and Shelanski ML: Caspase-2 redux. Cell Death Differ 10: 101-107, 2003.

9. Ridley AJ and Hall A: The small GTP-binding protein rho regulates the assembly of focal adhesions and actin stress fibers in response to growth factors. Cell 70: 389-399, 1992.

10. Hakuma N, Kinoshita I, Shimizu Y, Yamazaki K, Yoshida K, Nishimura $\mathrm{M}$ and Dosaka-Akita $\mathrm{H}$ : E1AF/PEA3 activates the Rho/Rho-associated kinase pathway to increase the malignancy potential of non-small cell lung cancer cells. Cancer Res 65: 10776-10782, 2005

11. Chan AY, Coniglio SJ, Chuang YY, Michaelson D, Knaus UG, Philips MR and Symons M: Roles of the Rac1 and Rac3 GTPases in human tumor cell invasion. Oncogene 24: 7821-7829, 2005.

12. Frisch SM, Vuori K, Ruoslahti E and Chan-Hui PY: Control of adhesion-dependent cell survival by focal adhesion kinase. J Cell Biol 134: 793-799, 1996.

13. Richardson A and Parsons T: A mechanism for regulation of the adhesion-associated protein tyrosine kinase pp125FAK. Nature 380: 538-540, 1996.

14. Bouzahzah B, Albanese C, Ahmed F, Pixley F, Lisanti MP, Segall JD, Condeelis J, Joyce D, Minden A, Der CJ, Chan A, Symons M and Pestell RG: Rho family GTPases regulate mammary epithelium cell growth and metastasis through distinguishable pathways. Mol Med 7: 816-830, 2001.

15. Mischel PS, Shai R, Shi T, Horvath S, Lu KV, Choe G, Seligson D, Kremen TJ, Palotie A, Liau LM, Cloughesy TF and Nelson SF: Identification of molecular subtypes of glioblastoma by gene expression profiling. Oncogene 22: 23612373, 2003.

16. Watanabe K, Tachibana O, Sata K, Yonekawa Y, Kleihues P and Ohgaki $\mathrm{H}$ : Overexpression of the EGF receptor and p53 mutations are mutually exclusive in the evolution of primary and secondary glioblastomas. Brain Pathol 6: 217-223, 1996.

17. Xue C, Liang F, Mahmood R, Vuolo M, Wyckoff J, Qian H, Tsai KL, Kim M, Locker J, Zhang ZY and Segall JE: ErbB3dependent motility and intravasation in breast cancer metastasis. Cancer Res 66: 1418-1426, 2006.

18. Muller-Tidow C, Diederichs S, Bulk E, Pohle T, Steffen B, Schwable J, Plewka S, Thomas M, Metzger R, Schneider PM, Brandts $\mathrm{CH}$, Berdel WE and Serve H: Identification of metastasisassociated receptor tyrosine kinases in non-small cell lung cancer. Cancer Res 65: 1778-1782, 2005.

19. Bernards A: Neurofibromatosis type 1 and Ras-mediated signaling: filling in the GAPs. Biochim Biophys Acta 1242: 43-59, 1995. 
20. Cichowski K and Jacks T: NF1 tumor suppressor gene function: narrowing the GAP. Cell 104: 593-604, 2001.

21. Ozawa T, Araki N, Yunoue S, Tokuo H, Feng L, Patrakitkomjorn S, Hara T, Ichikawa Y, Matsumoto K, Fujii K and Saya $\mathrm{H}$ : The neurofibromatosis type 1 gene product neurofibromin enhances cell motility by regulating actin filament dynamics via the Rho-ROCK-LIMK2-cofilin pathway. J Biol Chem 280: 39524-39533, 2005.

22. Feng L, Yunoue S, Tokuo H, Ozawa T, Zhang D, Patrakitkomjorn S, Ichimura T, Saya $\mathrm{H}$ and Araki N: PKA phosphorylation and 14-3-3 interaction regulate the function of neurofibromatosis type I tumor suppressor, neurofibromin. FEBS Lett 557: 275-282, 2004.

23. Corral T, Jimenez M, Hernandez-Munoz I, Perez DC I and Pellicer A: NF1 modulates the effects of Ras oncogenes: evidence of other NF1 function besides its GAP activity. J Cell Physiol 197: 214-224, 2003.

24. Ludwig HC, Akhavan-Shigari R, Rausch S, Schallock K, Quentin C, Bockermann V and Kolenda H: Expression of focal adhesion kinase (p125 FAK) and proline-rich tyrosine kinase 2 (PYK2/CAKb) in cerebral metastases, correlation with VEGF-R-, ecNOS III-labelling and morphometric data. Anticancer Res 20: 1419-1424, 2000.

25. Hu B, Jarzynka MJ, Guo P, Imanishi Y, Schlaepfer DD and Cheng SY: Angiopoietin 2 induces glioma cell invasion by stimulating matrix metalloprotease 2 expression through the alphavbeta1 integrin and focal adhesion kinase signaling pathway. Cancer Res 66: 775-783, 2006.

26. Scian MJ, Stagliano KE, Anderson MA, Hassan S, Bowman M, Miles MF, Deb SP and Deb S: Tumor-derived p53 mutants induce NF-kappaB2 gene expression. Mol Cell Biol 25: 10097-10110, 2005.

27. Viatour P, Bentires-Alj M, Chariot A, Deregowski V, de Leval L, Merville MP and Bours V: NF-kappa B2/p100 induces Bcl-2 expression. Leukemia 17: 1349-1356, 2003.

28. Reinholz MM, An MW, Johnsen SA, Subramaniam M, Suman VJ, Ingle JN, Roche PC and Spelsberg TC: Differential gene expression of TGF beta inducible early gene (TIEG), Smad7, Smad2 and Bard1 in normal and malignant breast tissue. Breast Cancer Res Treat 86: 75-88, 2004.

29. Tatenhorst L, Senner V, Puttmann S and Paulus W: Regulators of G-protein signaling 3 and 4 (RGS3, RGS4) are associated with glioma cell motility. J Neuropathol Exp Neurol 63: 210-222, 2004.
30. Zhao H, Jhanwar-Uniyal M, Datta PK, Yemul S, Ho L, Khitrov G, Kupershmidt I, Pasinetti GM, Ray T, Athwal RS and Achary MP: Expression profile of genes associated with antimetastatic gene: nm23-mediated metastasis inhibition in breast carcinoma cells. Int J Cancer 109: 65-70, 2004.

31. Kim LS, Huang S, Lu W, Lev DC and Price JE: Vascular endothelial growth factor expression promotes the growth of breast cancer brain metastases in nude mice. Clin Exp Metastasis 21: 107-118, 2004.

32. Olofsson B, Korpelainen E, Pepper MS, Mandriota SJ, Aase K, Kumar V, Gunji Y, Jeltsch MM, Shibuya M, Alitalo K and Eriksson U: Vascular endothelial growth factor B (VEGF-B) binds to VEGF receptor-1 and regulates plasminogen activator activity in endothelial cells. Proc Natl Acad Sci USA 95: 11709-11714, 1998.

33. Luttun A, Tjwa $M$ and Carmeliet P: Placental growth factor (PIGF) and its receptor Flt-1 (VEGFR-1): novel therapeutic targets for angiogenic disorders. Ann NY Acad Sci 979: 80-93, 2002.

34. Sun Y, Jin K, Childs JT, Xie L, Mao XO and Greenberg DA: Vascular endothelial growth factor-B (VEGFB) stimulates neurogenesis: evidence from knockout mice and growth factor administration. Dev Biol 289: 329-335, 2006.

35. Woo IS, Park MJ, Byun JH, Hong YS, Lee KS, Park YS, Lee JA, Park YI and Ahn HK: Expression of placental growth factor gene in lung cancer. Tumour Biol 25: 1-6, 2004.

36. Nomura M, Yamagishi S, Harada S, Yamashima T, Yamashita J and Yamamoto $\mathrm{H}$ : Placenta growth factor (PlGF) mRNA expression in brain tumors. J Neurooncol 40: 123-130, 1998

37. Hatanaka H, Oshika Y, Abe Y, Yoshida Y, Hashimoto T, Handa A, Kijima H, Yamazaki H, Inoue H, Ueyama Y and Nakamura M: Vascularization is decreased in pulmonary adenocarcinoma expressing brain-specific angiogenesis inhibitor 1 (BAI1). Int J Mol Med 5: 181-183, 2000.

38. Linderholm BK, Lindahl T, Holmberg L, Klaar S, Lennerstrand J, Henriksson R and Bergh J: The expression of vascular endothelial growth factor correlates with mutant p53 and poor prognosis in human breast cancer. Cancer Res 61: 2256-2260, 2001 . 